\title{
Simulating the Performance of Daylight-Linked Control Systems
}

\author{
Laura Bellia ${ }^{1}$, Francesca Fragliasso ${ }^{1}$ \\ ${ }^{1}$ Department of Industrial Engineering of the University of Naples "Federico II", Naples, Italy
}

\begin{abstract}
Despite the progress in daylight modelling determined by the development of dynamic daylight calculation software, the simulation of daylight-linked control systems (DLCSs) does not provide reliable results, since available calculation software are not able to account for all the factors influencing DLCSs performance. The paper presents a new tool called DET (DLCSs Evaluation Tool) able to simulate DLCSs and to evaluate the energy saving they provide. A simple case-study is used to compare DET results with those provided by DIVA for Rhino, demonstrating that DET generally calculates higher consumptions than DIVA.
\end{abstract}

\section{Introduction}

Daylight-linked control systems (DLCSs) represent a useful strategy to save energy due to electric lighting (Dubois and Blomsterberg 2011) and at the same time to improve occupants' wellbeing: Their use allows optimizing daylighting, positively affecting people's visual and non-visual comfort (Boyce et al. 2003).

Despite the progress in daylight modelling determined by dynamic daylight simulations introduction, the way DLCSs are modelled is not very reliable. Factors affecting their performance are a lot (Bellia et al. 2016) (photosensor characteristics, control strategy, calibration conditions, lighting systems characteristics) and available software do not account for all of them.

Currently, DLCSs functioning is generally simulated by means of the same software used to dynamically simulate daylight availability in indoor environments. In this field, the most accredited software is surely Daysim, a validated software based on RADIANCE engine. In Daysim, the user divides the work-plane in different control zones and, for each one of them, sets the control strategy and the lighting system characteristics. For each control zone, the software dynamically calculates the luminaires light output necessary to perfectly integrate daylight, the related absorbed power and the corresponding energy consumptions, assuming an hourly or sub-hourly time step. The most significant approximations of this software are two: luminaires light output is evaluated starting from illuminance at the work-plane and not from photosensor detections; the calibration procedure is completely neglected.

The same calculation procedure is used in DIVA: a highly optimized daylighting modelling plug-in for
Rhinoceros based on Daysim engine. Differently from Daysim, DIVA has another limitation: it allows simulating daylight availability exclusively considering a 1-hour time step, but previous studies (Littlefair 2001, Bellia et al. 2018) underlined that brief-time daylight oscillations strictly influence DLCSs functioning.

Another DLCSs simulation tool based on Daysim is the modified JAVA GUI for Daysim developed at Penn State University by Mistrick. It introduces interesting novelties compared with Daysim, since it is able to model photosensors characteristics and to account for different control algorithms. However, it is not perfectly compatible with Windows operating systems.

Rogers developed an Excel Macro called SPOT (Sensor Placement + Optimization Tool) (Rogers 2006) useful during DLCSs design process to define control strategies, to choose photosensors from a database of commercially available products and to establish their location. Furthermore, the software is able to simulate the calibration phase of different control typologies (switching, stepped and dimming ones). Its limitations are the following: daylight availability modelling is simplified and only spaces characterized by simple geometry can be modelled.

Since each available software is based on different calculation procedure, the use of a software or another determines a different evaluation of the related energy consumptions. For example, Doulos et al. (2005) compared SPOT and Daysim and they found that energy consumptions obtained by means of SPOT are $15 \%$ lower than those obtained by means of Daysim.

Based on these premises, researchers proposed different methodologies to overcome the weak points of the available software. Some of these studies suggest the use of quick and simplified methods, alternative to dynamic daylight simulations, that can be useful especially in the first phase of the design process. In this context the study by Lo Verso et al. (2014) are interesting. Among the simplified calculation models, the one proposed by the European standard EN 15193-1:2017 has to be considered as well.

On the other hand, other studies proposed strategies to perfect the dynamic calculation methods overcoming the above-mentioned limitations. For example, one of the most debated issue is photosensors modelling, accounting for spatial and spectral responses (Doulos et al. 2008b, Ehrlich et al. 2002). Other researches (Doulos 
et al. 2008a, Roisin et al. 2008) focused on the lighting systems characteristics, deepening issues connected to the ballast dimming response function, i.e. the curve relating light output and absorbed power.

This paper presents a simulation tool called DET (DLCSs Evaluation Tool) developed by Bellia and Fragliasso (2017). It can be used to post-process dynamic daylight simulation results obtained by using software like Daysim or DIVA, in order to simulate DLCSs performance, accounting for some aspects not considered by the currently available calculation tools. The work is divided in two parts. In the former one, after a brief description of DET, the calculation procedures used by DIVA and by DET are described and compared. In the latter, the two tools are used to simulate the functioning of closed-loop proportional dimming systems in a simple case study and obtained results are compared.

\section{A little focus on DET}

DET is an Excel worksheet with macro and it is conceived to be a sort of external plug-in for dynamic daylight simulation software. It consists in three mainly screens that users can easily navigate, moving from one to another (see for example Figure 1). The first screen allows input data to be inserted and the specific control algorithm to be selected. In the second one the setting parameters referred to the chosen control algorithm must be added. Finally, the third screen shows the results.

As it can be seen in Figure 1, to use DET, users must simply insert the parameters indicated in the blue cells, writing them in the corresponding grey cells. Specifically, in the first screen, data to insert in columns C, D, E and F must be copied and pasted from the output files of the dynamic daylight simulations software. Once this is done, users must push one of the buttons named as the corresponding control algorithm (open-loop and closed-loop switching, open-loop and closed-loop stepped, open-loop dimming, closed-loop integral reset and proportional). The second screen (see Figure 2), different for each algorithm, must be used to insert the parameters necessary to simulate the calibration of the DLCS. From the same screen, users can come back to the previous section, if they got wrong in choosing the control algorithm. Once all the parameters are set, the "Simulate control system functioning" allows running the simulation. At this point, all the results will automatically appear in the last screen in the form of graphs similar to those reported in Results section (see Figure 5) together with the numerical values which graphs are referred to.

\section{Comparison between DIVA and DET}

In both DIVA and DET, modelling of DLCSs functioning is based on the results of a previously performed dynamic daylight simulation, aiming at obtaining annual illuminance trends at the work-plane. In DIVA, the two calculation phases are strictly connected, since the same software is used to carry out both of them. On the contrary, when using DET, the daylight simulation results obtained by means of DIVA or another calculation software must be transferred in DET.

Despite it is different, the DLCSs modelling procedure can be divided in four different steps for both software:

1. Setting of calculation parameters referred to the control system;

2. Definition of the photosensor location and of the work-plane control points (i.e. those points where specific lighting conditions must be guaranteed);

3. Calculation of the luminaires light output $(\delta)$ (expressed as the percentage ratio of the emitted luminous flux to the total one), necessary to integrate daylight and achieve the required task illuminance at the control points;

4. Calculation of the absorbed power based on the obtained light output.

Considering that the calculation approach is dynamic, steps 3 and 4 are repeated for each value of work-plane daylight illuminance previously obtained by means of the dynamic daylight simulations.

\section{DIVA - Step 1: Setting of calculation parameters}

In DIVA the DLCSs dimming system functioning can be simulated by adding the "Photosensor controlled dimming" control to the lighting system. For this control typology, the corresponding calculation parameters are:

- Lighting Power (LP) [W]: the power absorbed by the lighting system when luminaires are on and the light output is $100 \%$;

- Lighting Setpoint (LS) [lx]: the target illuminance prescribed by regulations;

- Ballast Loss Factor (BLF) [\%]: the percentage of peak energy used by a dimming system, when it is dimmed down at $1 \%$.

- Standby Power (SP) [W]: the amount of energy absorbed by some automatically lighting systems even when the system is "off".

\section{DIVA - Step 2: Definition of work-plane and photosensor location}

In DIVA there is no difference between the photosensor and the work-plane location, meaning that the DLCS functioning is simulated considering that the photosensor is located at the work-plane and, consequently, the light it detects is the same received by the work-plane. The user has to select one or more points belonging to the work-plane calculation grid used for dynamic daylight simulations, then the software models the DLCS, considering that the daylight illuminance at the workplane will be perfectly integrated by means of electric light. If more than one point is selected, luminaires will be dimmed, in order to guarantee the $L S$ at the most disadvantaged point, i.e. the point receiving less daylight.

\section{DIVA - Step 3: Light output calculation}

For each hourly data $t$, the luminaires light output, $\delta_{\mathrm{t}}$, is evaluated as it follows: 
$\delta_{t}= \begin{cases}0.01 & \text { if } E_{d l, t} \geq L S \\ \frac{\left(L S-E_{d l, t}\right)}{L S} & \text { if } E_{d l, t}<L S\end{cases}$

Where $E_{d l, t}$ is the daylight illuminance at the most disadvantaged of the points selected by the user.

\section{DIVA - Step 4: Absorbed power calculation}

In DIVA, the calculation of the absorbed power is divided in two phases. In the former one, starting from $\delta_{t}$, the Power Ratio $\left(P R_{t}\right)$ is calculated. $P R_{t}$ is the percentage ratio of the absorbed power at the time $t\left(P_{t}\right)$ to the total power of the system. To calculate $P R_{t}$, it is assumed that there is a linear relationship between the light output and the absorbed power. The slope of the straight line describing the relation can be defined considering that when $\delta$ is maximum, i.e. it is $100 \%$, the corresponding power ratio is $100 \%$, whereas, when $\delta$ is minimum, i.e. it is $1 \%$, the power ratio is equal to the Ballast Loss Factor. So, at each time t, the power ratio is:

$P R_{t}=\left\{\begin{array}{l}B L S \\ \frac{1-B L F}{0.99} \cdot \delta_{t}+\frac{B L F-0.01}{0.99} \text { if } \delta_{t}=0.01 \\ \text { if } \delta_{t}>0.01\end{array}\right.$

In the latter phase, the absorbed power is calculated starting from $P R_{t}$ as:

$P_{t}=P R_{t} \cdot(L P+S P)$

So, the software considers that both the LP and the SP are dimmed as a function of the available daylight.

As regards the output, the software provides exclusively the annual trend of the $P R_{t}$ and the annual energy consumption expressed in $\mathrm{kWh}$.

\section{DET - Steps 1 and 2: Setting of calculation parameters and definition of work-plane and photosensor location}

As it was previously reported, DET is conceived to be a sort of external plug-in of dynamic daylight simulation software. So, before setting the calculation parameters specifically referred to the control system, the results of the previously performed dynamic daylight simulations must be copied and pasted in the first software screen (Figure 1). Here the user has to insert the following data:

- $E_{\text {task }}$ : the illuminance required by regulations;

- Time step: the time step of the dynamic daylight simulations. It can be 1 hour (like in DIVA) or 1 minute. The 1-minute time step is considered since DET can be used coupled not only with DIVA, but also with other software like Daysim, able to provide as output sub-hourly data. As an alternative, the user can insert results of measured daylight data as well;

- Amount of days: number of simulation days;

- Detections per day: number of data obtained by means of the simulation each day.

Then in the columns from $\mathrm{C}$ to $\mathrm{F}$, the user must copy simulations results in the following order:

- Day in column C;

- Time in column D;

- $E_{d l}$ in column E;

- $S_{d l}$ in column F.
$E_{d l}$ is the daylight work-plane illuminance obtained by means of simulations. The user can insert illuminance values referred to the most disadvantaged point of the calculation grid or to the average values of all the calculation points. $S_{d l}$ is the photosensor signal. Differently from DIVA, in DET the light output is evaluated according to the detections of the photosensor and not to the work-plane illuminances. For this reason, when using DET, during the previous dynamic daylight simulations, it is necessary to set both a calculation grid located at the work-plane and a calculation point at the location of the photosensor. Illuminances at this point can be assumed coincident with the photosensor detections and copied in D column.

After dynamic daylight simulation results have been inserted, the user chooses the control algorithm by clicking the corresponding button. In this way he activates the following screen (see Figure 2). In DET, the control algorithm is in-depth modelled, accounting for the calibration phase. For example, for closed-loop dimming systems (considered in this application) the control functioning is simulated according to the algorithm reported in Figure 2. It considers that luminaires are continuously regulated from a maximum light output to a minimum one, without being completely switched-off. The slope of the control straight line is evaluated defining two points during the calibration. The first one is set night-time, when daylight is absent, the light output is the maximum one and the photosensor detects exclusively electric light. The second set-point is defined when daylight is such that the light output necessary to integrate daylight is slightly above the minimum one (Di Laura et al. 2011). To properly set the control algorithm, the following parameters are needed:

- $\delta_{\max }[\%]$ : the maximum light output of the luminaires;

- $\delta_{t c}[\%]$ : the light output at the calibration. It depends on daylight conditions occurring at the calibration;

- $\delta_{\min }[\%]:$ the minimum light output which luminaires can be dimmed down at;

- $S_{e l, \delta \max }$ : electric light component of the photosensor signal at night-time calibration;

- $S_{d l, t c}$ : daylight signal at the photosensor at day-time calibration;

- $E_{e l, \delta \max }[\mathrm{lx}]$ : electric light work-plane illuminance at night-time calibration;

- $E_{d l, t c}[\mathrm{~lx}]$ : daylight illuminance at the work-plane at day-time calibration;

- $P_{100 \%}[\mathrm{~W}]:$ power absorbed by all luminaires when they are on at $100 \%$;

- $P_{\text {smin }}$ [W]: power absorbed by all luminaires when they are on at the minimum light output;

- $P_{\text {stand-by }}[\mathrm{W}]$ : power absorbed by all luminaires in stand-by conditions;

- $P_{\text {other }}[\mathrm{W}]$ : power absorbed by auxiliary devices such as photosensors and controllers.

Differently from DIVA the minimum and maximum light output are set by the user and not chosen by default. 


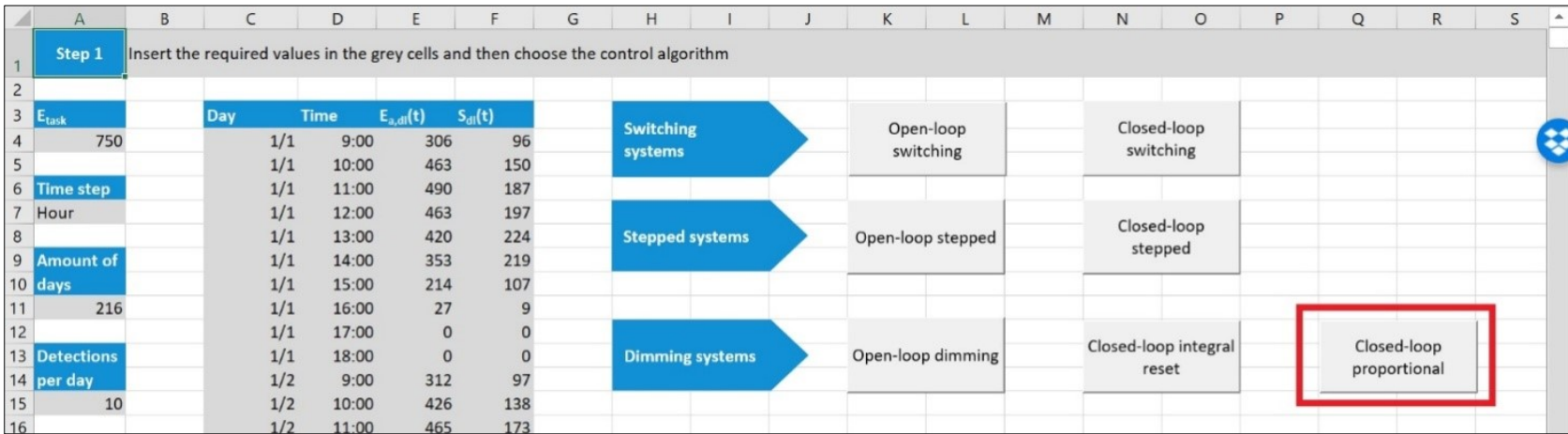

Figure 1: DET first screen

This allows accounting two factors: 1) the minimum light output can be different depending on the chosen light source and it is not necessarily equal to $1 \%$; 2 ) the use of DLCSs can be associated to the luminance maintenance control strategy. Indeed, lighting systems are generally over-dimensioned to consider the luminous flux decay over time. So, at the beginning of their life cycle luminaires can be dimmed to save energy. This means that, when setting the DLCS, the maximum light output must be lower than $100 \%$.

Parameters referred to daylight data at calibration $\left(S_{d l, t c}\right.$ and $\left.E_{d l, t c}\right)$ can be inferred from results of dynamic daylight simulations, choosing a daylight condition proper for the calibration, according to practice prescriptions (Di Laura et al. 2011). On the other hand, parameters referred to electric light $\left(S_{e l, \delta \max }\right.$ and $\left.E_{e l, \delta \max }\right)$ can be obtained by means of DIVA itself, or by means of another electric light simulation software.

\section{DET - Step 3: Light output calculation}

For each hourly or sub-hourly data $t$, considering closedloop dimming systems, the luminaires light output is evaluated as it follows:

$\delta_{t}=\left\{\begin{array}{l}\frac{m \cdot S_{d l, t}+q}{1-m \cdot S_{e l, 100 \%}} \text { if } S_{d l, t} \leq S_{d l, l i m} \\ \delta_{\min } \text { if } S_{d l, t}>S_{d l, l i m}\end{array}\right.$

Where:

$m=\frac{\delta_{t c}-\delta_{\max }}{S_{t c}-S_{e l, \delta \max }}$

$q=\frac{S_{t c} \cdot \delta_{\max }-S_{e l, \delta \max } \cdot \delta_{t c}}{S_{t c}-S_{e l, \delta \max }}$

$S_{d l, l i m}=\frac{\delta_{\min }\left(1-m \cdot S_{e l, 100 \%}\right)-q}{m}$

$S_{d l, t}$ is the daylight component of photosensor signal at each time $t$, inferred by the software from column $F$ in the first screen. $S_{t c}$ is the total photosensor signal at the day-time calibration (daylight component plus electric light component) and it is calculated as:

$S_{t c}=S_{d l, t c}+\delta_{t c} \cdot S_{e l, 100 \%}$

$S_{e l, 100 \%}$ is the electric light component of the photosensor signal when luminaires are on at $100 \%$ and it is:

$S_{e l, 100 \%}=\frac{S_{e l, \delta \max }}{\delta_{\max }}$

\section{DET - Step 4: Absorbed power calculation}

In DET, whatever control algorithm is chosen, at each time $t$, the absorbed power $\left(P_{t}\right)$ is:

$P_{t}=P_{\text {lum.t }}+P_{\text {other }}$

Where $P_{\text {lum.t }}$ is the power absorbed by the luminaires and $\mathrm{P}_{\text {other }}$ is that absorbed by the photocell and other auxiliary devices. In turn, $\mathrm{P}_{\text {lum.t }}$ can be evaluated assuming a linear relationship between the light output and the absorbed power and considering that, when the light output is equal to $\delta_{\min }$, the absorbed power is equal to $P_{\delta \min }$, when it is equal to $100 \%$ the power is $P_{100 \%}$. $\mathrm{P}_{\text {lum.t }}$ is evaluated as:

$P_{\text {lum.t }}=\left\{\begin{array}{l}m_{P} \cdot \delta_{t}+q_{P} \text { if luminaires are on } \\ P_{\text {stand }- \text { by }} \text { if luminaires are of } f\end{array}\right.$

Where:

$$
\begin{aligned}
& m_{P}=\frac{P_{100 \%}-P_{\delta \min }}{1-\delta_{\min }} \\
& q_{P}=\frac{P_{\delta \min }-\delta_{\min } \cdot P_{100 \%}}{1-\delta_{\min }}
\end{aligned}
$$

So, in DET only the luminaires power is dimmed and it is considered that the stand-by power and the power of other components are steady rates of the power.

As regards the output, DET provides the energy consumptions in $\mathrm{kWh}$ and the trends on time varying of light output, absorbed power, electric light illuminance at the work-plane and total illuminance at the workplane (daylight plus electric light).

\section{Case study description}

Dynamic daylight simulations were performed by means of DIVA referred to a typical sidelit office $4 \mathrm{~m} \cdot 4 \mathrm{~m}$ wide and $3 \mathrm{~m}$ high. The room dimensions are reported in Figure 3. Simulations were repeated 8 times considering that the office was located in two cities characterized by different weather conditions, Naples (Latitude $40^{\circ} 51^{\prime} 22$ $\mathrm{N}$, Longitude $14^{\circ} 14^{\prime} 47 \mathrm{E}$ ) and Berlin (Latitude $52^{\circ} 31^{\prime}$ $27 \mathrm{~N}$, Longitude $13^{\circ} 24^{\prime} 37 \mathrm{E}$ ), and assuming that for each city the window was oriented according to the four main cardinal points in turn. It was considered that the office was located at the second floor of a three-story building and that a building of the same height was positioned in front of it at a distance of about $15 \mathrm{~m}$. 


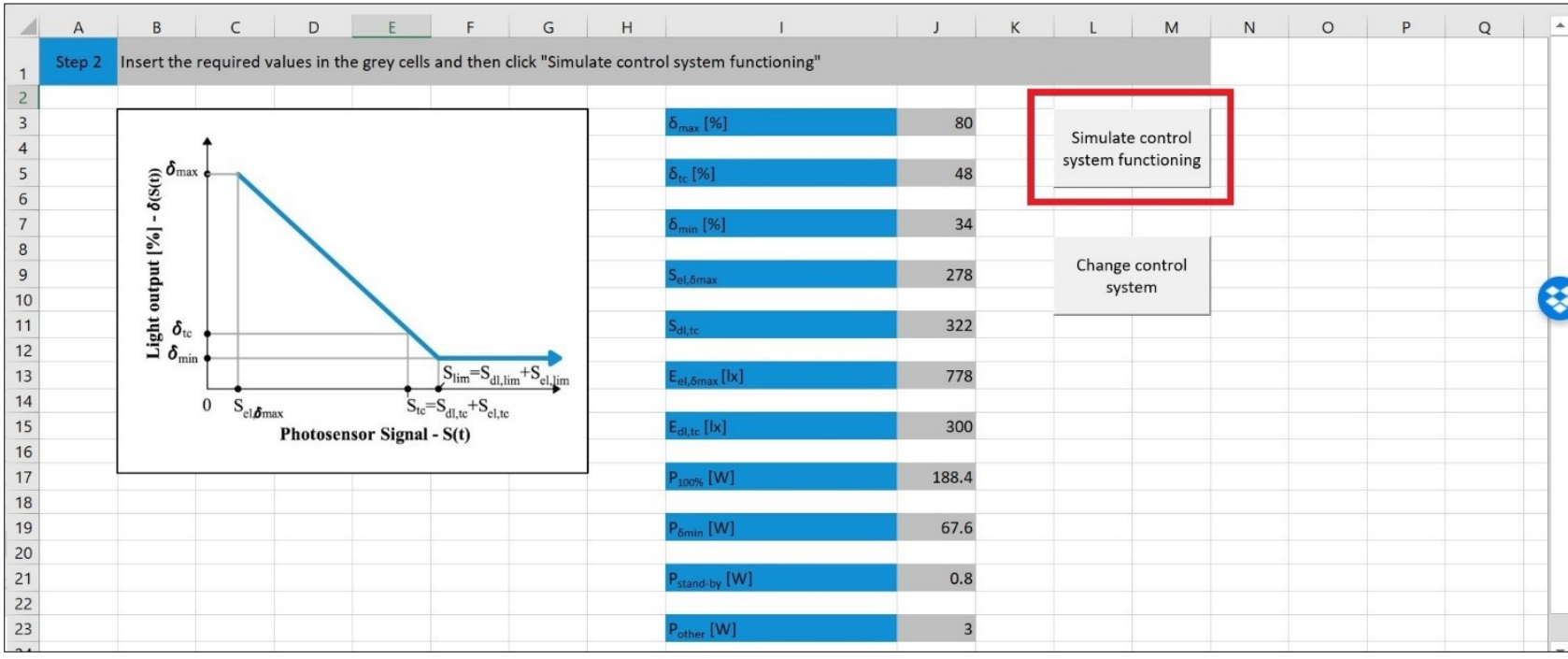

Figure 2: Closed-loop proportional dimming setting screen

The following reflectances were used for architectural surfaces: 0.50 - interior walls, 0.80 - ceiling, $0.20-$ floor, 0.50 - furniture, 0.50 - window frame, $0.35-$ exterior walls, 0.20 - outside ground. The visual transmittance of the window glazing was 0.8 . As for shading devices, the DIVA "conceptual shading", modelled according the Lightswitch algorithm (Reinhart 2004) was used for east, south and west windows. This algorithm considers that an ideal shading system is activated when direct irradiance at the work-plane is higher than $50 \mathrm{~W} / \mathrm{m}^{2}$. When the shading is activated the direct component of daylight is completely shielded and the diffuse one is reduced of $75 \%$. The weather data file used for simulations were IWEC ones and they were downloaded from the Energy Plus web site. It was considered that the office was occupied from Monday to Friday, from 9:00 to 18:00 and that daylight-saving time ranged from April the $1^{\text {st }}$ to October the $31^{\text {st }}$. Calculation parameters were set according to (Reinhart 2006) and they were: ambient bounces 7, ambient division 1500 , ambient sampling 100, ambient resolution 300, ambient accuracy 0.05 . Illuminance values were calculated both for a calculation grid located at the work-plane (points from $\mathrm{W} 1$ to $\mathrm{W} 6$ in Figure 3) and at a point positioned at the ceiling and simulating the photosensor ( $\mathrm{P}$ point in Figure 3). By using DIALux software a lighting system was designed considering that the performed visual task was "technical drawing". To perform this task, according to the EN 12464-1:2011, an average illuminance value equal to $750 \mathrm{~lx}$ and a uniformity value of 0.7 have to be achieved.

The chosen typology of luminaire was a recessed dimmable LED light source previously tested at the Photometry and Lighting Laboratory of the Department of Industrial Engineering of the University of Naples Federico II (Italy). Specifically, in the laboratory measurements of absorbed power and illuminance were performed by means of an electronic power meter connected to the fuse box of the laboratory and by a Konica Minolta T10A illuminance meter, located under the luminaire at the horizontal plane and at a distance from the floor equal to $0.75 \mathrm{~m}$. Measurements were repeated varying the luminous flux from the minimum to the maximum by means of a DALI controller. It was found that there was a linear relationship between light output and absorbed power and that the minimum light output was $34 \%$ (the work-plane illuminance varies from $115 \mathrm{~lx}$ to $338 \mathrm{~lx}$ ). Moreover, when the light output was $100 \%$ the absorbed power was $47.1 \mathrm{~W}$, whereas when the light output was $34 \%$, the power was $16.9 \mathrm{~W}$, i.e. the $36 \%$ of the total power. To fulfil prescriptions, considering a 0.8 maintenance factor, four luminaires (total power $188.4 \mathrm{~W}$ ) were necessary (see Figure 3), guaranteeing at the desk an average illuminance equal to $816 \mathrm{~lx}$ and a uniformity value equal to 0.96 .

Based on the daylight illuminance trends obtained by means of the dynamic daylight simulations, by knowing the characteristic of the lighting system, the functioning of the closed-loop dimming system was simulated with both DIVA and DET. Tables 1 and 2 report calculation parameters related to the control system for DIVA and DET respectively.

Table 1: DIVA control calculation parameters

\begin{tabular}{|c|c|}
\hline Calculation Parameter & Corresponding value \\
\hline Lighting Power (LP) & $188.4 \mathrm{~W}$ \\
\hline Lighting Setpoint (LS) & $750 \mathrm{~lx}$ \\
\hline Ballast Loss Factor (BLF) & $4 \%$ \\
\hline Standby Power (SP) & $3 \mathrm{~W}$ \\
\hline
\end{tabular}

Table 2: DET control calculation parameters

\begin{tabular}{|c|c|}
\hline Calculation Parameter & Corresponding value \\
\hline$\delta_{\max }$ & $100 \%$ \\
\hline$\delta_{\min }$ & $34 \%$ \\
\hline$S_{e l, \delta \max }$ & $355 \mathrm{~lx}$ \\
\hline$E_{e l, \delta \max }$ & $1020 \mathrm{~lx}$ \\
\hline $\mathrm{P}_{100 \%}$ & $188.4 \mathrm{~W}$ \\
\hline $\mathrm{P}_{\delta \min }$ & $67.6 \mathrm{~W}$ \\
\hline $\mathrm{P}_{\text {stand-by }}$ & $0.8 \mathrm{~W}$ \\
\hline $\mathrm{P}_{\text {other }}$ & $3 \mathrm{~W}$ \\
\hline
\end{tabular}




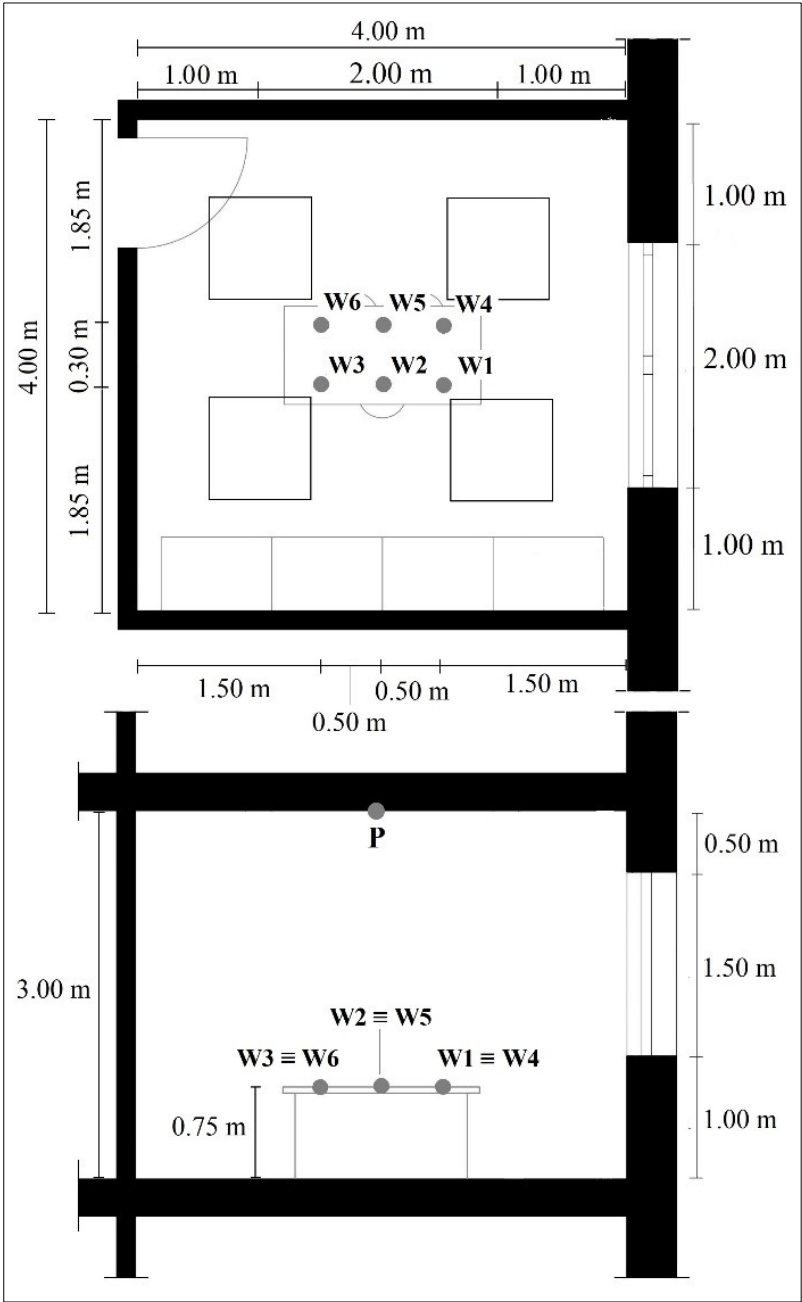

Figure 3: Office measured plan and section

For DIVA simulations, all the work-plane calculation points were selected as control points. Laboratory measurements demonstrated that luminaires cannot be dimmed under the $34 \%$, however DIVA considers by default that the luminous flux can be reduced till the $1 \%$. For this reason, the BLF (i.e. the percentage of power that luminaires would absorb if they were able to reach the $1 \%$ of light output) was obtained considering the linear relation between power and light output observed in the laboratory. Moreover, it must be noticed that, despite the name, the DIVA "Stand-by power" calculation parameter cannot be considered an actual stand-by power, indeed, according to the calculation procedure (see the equation (3)), it is not the power absorbed when luminaires are off. For this reason, the $3 \mathrm{~W}$ value (i.e. the value used in DET for the $P_{\text {other }}$ ) was attributed to the stand-by parameter in DIVA. On the contrary, in DET, the stand-by power value was that measured in the Laboratory ( $0.2 \mathrm{~W}$ per luminaire). Electric light illuminance values at the work-plane and at the photosensor useful to simulate the night-time calibration were calculated by means of DIALux. It was considered that the maximum light output was $100 \%$, in order to make results comparable with those provided by DIVA.
Table 3: DET day-time calibration parameters

\begin{tabular}{|c|c|c|c|}
\hline & $\boldsymbol{E}_{\boldsymbol{d l}, \boldsymbol{t} \boldsymbol{c}}[\mathbf{l x}]$ & $\boldsymbol{S}_{\boldsymbol{d l}, \boldsymbol{t} \boldsymbol{c}}[\mathbf{l x}]$ & $\boldsymbol{\delta}_{\boldsymbol{t c}}[\mathbf{\%} \boldsymbol{\mathbf { o }}]$ \\
\hline Naples East & 377 & 321 & 40 \\
\hline Naples South & 350 & 210 & 43 \\
\hline Naples West & 386 & 310 & 39 \\
\hline Naples North & 342 & 206 & 43 \\
\hline Berlin East & 356 & 292 & 42 \\
\hline Berlin South & 188 & 350 & 43 \\
\hline Berlin West & 189 & 335 & 45 \\
\hline Berlin North & 196 & 362 & 42 \\
\hline
\end{tabular}

As for the day-time calibration, for each case study a couple of work-plane illuminance and photosensor signal was inferred by the dynamic daylight simulation results (see Table 3) and the corresponding light output value was calculated. For DET simulations, when uploading dynamic daylight simulation results (Figure 1), the average value of the daylight illuminances at the six calculation points was inserted in the E column.

\section{Results}

Figure 4 reports annual energy consumption in $\mathrm{kWh}$ for both cities calculated by means of DIVA and DET related to each orientation. As it was easy to predict, DET always determines higher consumptions compared with DIVA. This happens because DIVA considers that luminaires can be dimmed till the $1 \%$, whereas DET uses $34 \%$ as minimum light output. It must be observed that the percentage difference between consumptions evaluated with the two software (indicated on the graphs) depends on the specific case. It ranges from $1.54 \%$ (Berlin-East orientation) to $14.87 \%$ (Naples North orientation). By the data observation it can be concluded that the higher the daylight availability is, the more the percentage difference between the two software increases. North orientation is characterized by the highest daylight availability and consequently lower energy consumption, since for this configuration, it is never necessary to use shading. On the contrary, given the occupancy scheduling and the necessity to use shading system, the east orientation is more disadvantaged in terms of daylight availability. This determines higher consumptions. When daylight availability is significant and work-plane daylight illuminances are generally high and close to the required task illuminance, the minimum light output has a significant weight in determining consumptions. Indeed, the hours amount during which luminaires are on at the minimum light output is high. This explains the results trend. It must be underlined that, even when the percentage differences between the two software are low, the modelled power trends can be very different, meaning that the luminaires light output and the related lighting conditions are different. This can be observed in Figure 5, representing the power trends corresponding to June and December and referred to the two limit cases for which the minimum and the maximum percentage differences were observed (Berlin-East and NaplesNorth). It can be noticed that the differences between the power trends modelled by the two software are not due exclusively to the minimum light output setting. 


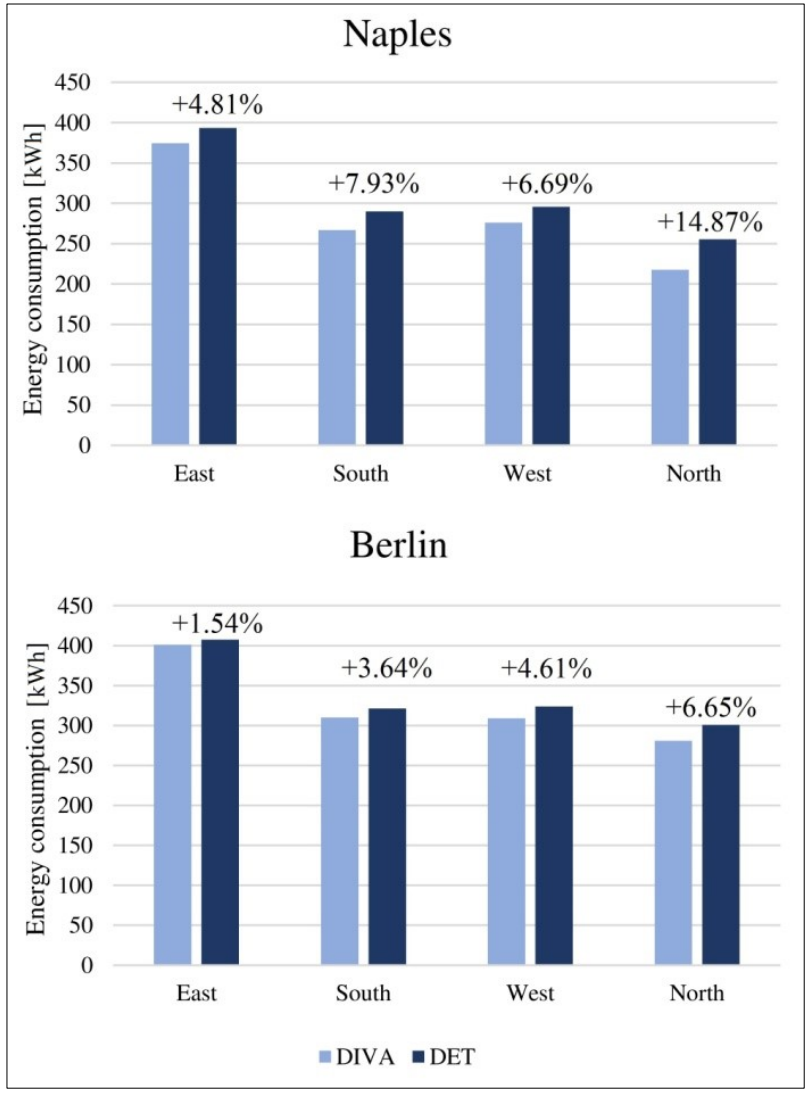

Figure 4: Energy consumption due to lighting system

Indeed, the shifts can be observed for power levels higher than $P_{\delta \min }$ (i.e. $67.6 \mathrm{~W}$ ) as well. In this case, the differences depend on the fact that DET calculates the light output according to the photosensor detections and not according to the work-plane illuminances. Finally, it must be underlined that the differences between the two software depends on the considered month as well. On December, differently from June, the trends are very similar. This means that the photosensor detections can be more or less representative of the work-plane illuminance conditions depending on the daylight availability, and specifically on the daylight distribution in the studied environment, that in turn depends on the outdoor weather conditions. The lower daylight availability is and the higher the skylight incidence in determining work-plane illuminances is, the more reliable the photosensor detections are. In these conditions the calculation approximations due to the fact that DIVA models the control functioning directly starting from work-plane illuminances are less significant, and the trends obtained using the two software are more similar.

\section{Conclusions}

The paper analysed the way two software, DIVA and DET, model the functioning of closed-loop dimming control systems, comparing the results obtained related to 8 simple case-studies. It was found that DET always provides energy consumptions values higher than DIVA. This is due to two calculation simplifications of DIVA software, overcome by DET: 1) differently from DET, DIVA considers that luminaires can be always dimmed

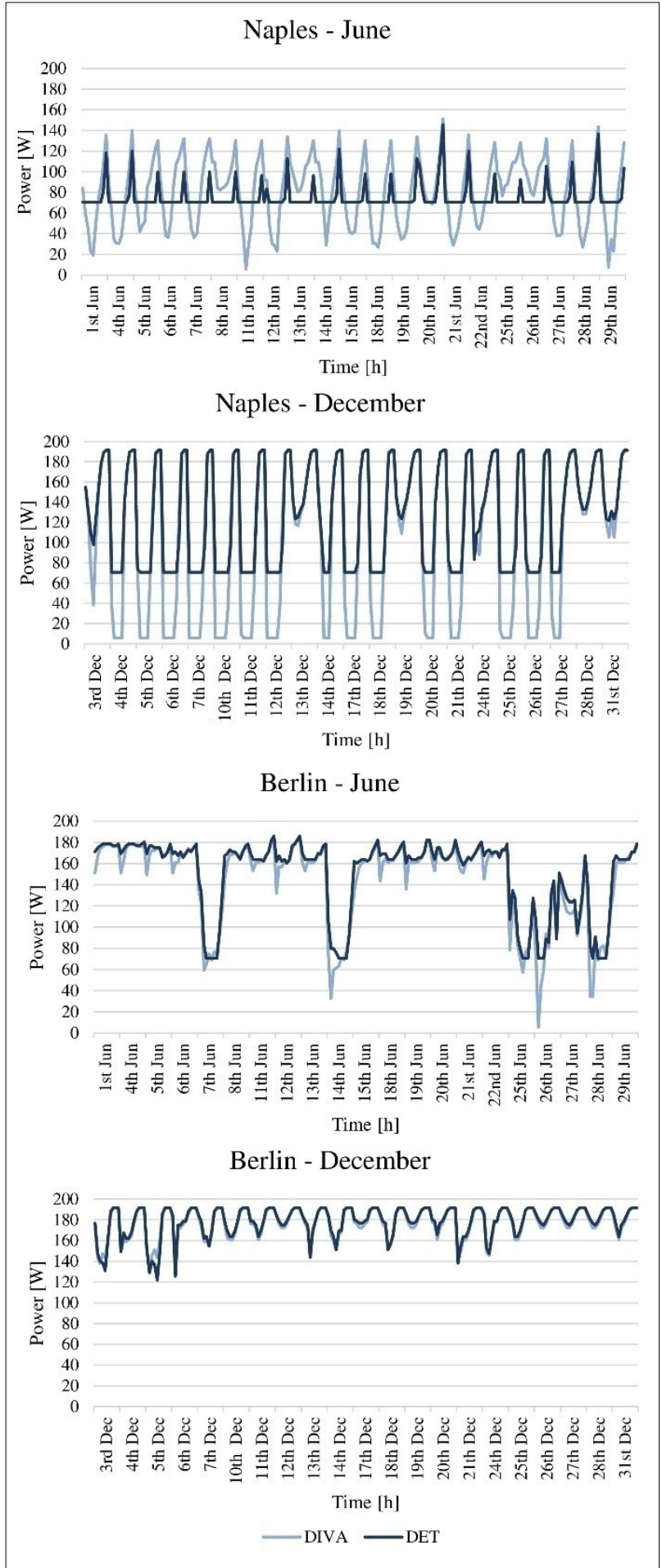

Figure 5: Power trends during June and December related to Naples - North and Berlin - East

down till a minimum value equal to $1 \%$ of the total emitted flux, that is not true for all the light sources; 2) DIVA calculates the power absorbed by the luminaires starting from work-plane illuminances and not from photosensor detections. The observed differences have not the same weight for each observed case-study. Specifically, it was observed that the higher the daylight availability is, the more divergent the results provided by the two software are. This is due to the incidence of the minimum luminaires light output setting. Moreover, it 
was demonstrated that, even when the annual energy consumptions are similar, the corresponding absorbed power trends can be very different. This depends on the fact that DET, differently from DIVA, calculates the control system functioning starting from the photosensor detections. DIVA assumes that the integration between daylight and electric light is always perfect, since it considers that the photosensor is located at the workplane. This determines that, when electric light is needed, the sum of daylight and electric light illuminances at the work-plane is always equal to the task. On the contrary, when the photosensor is realistically located, the integration is not perfect. DET allows to evaluate this issue, providing, beyond the power trends, the illuminance trends at the work-plane. This allows assessing if during its operating life the control system is able to integrate daylight, or it determines illuminance levels not adequate to fulfil regulation prescriptions. Moreover, it must be underlined that DET is composed of two calculation modules: the simulation one (described in the paper) and the evaluation one. The evaluation one contains the calculation procedures to assess the performance of DLCSs based on their capability in integrating daylight expressed by means of specific performance parameters DIA (Daylight Integration Adequacy), LD\% (Percentage Light Deficit), ILE\% (Percentage Intrinsic Light Excess) and $\mathrm{LW}_{\%}$ (Percentage Light Waste) proposed by Bellia and Fragliasso (2017). On the contrary, in DIVA the evaluation of the performance is exclusively done by evaluating the energy consumptions. Finally, contrarily to DIVA, by means of DET different control systems typologies can be simulated (open-loop switching, stepped and dimming systems and closed-loop switching, stepped, integral reset and proportional dimming systems). Further studies are necessary to validate DET, by means of comparisons with real control systems to evaluate the reliability of the obtained results.

\section{References}

Bellia, L. \& Fragliasso, F. (2017). New parameters to evaluate the capability of a daylight-linked control system in complementing daylight. Building and Environment, 123, 223-242.

Bellia, L., Fragliasso, F. \& Riccio, G. (2018). Daylight fluctuations effect on the functioning of different daylight-linked control systems. Building and Environment, 135, 162-193.

Bellia, L., Fragliasso, F. \& Stefanizzi, E. (2016). Why are daylight-linked controls (DLCs) not so spread? A literature review. Building and Environment, 106, 301-312.

Boyce, P., Hunter, C. and Howlett, O. 2003. The benefits of daylight through windows. Troy, New York: Rensselaer Polytechnic Institute.

Di Laura, D. L., Houser, K. H., Mistrick, R. G. \& Steffy, G. R. (2011). Lighting controls in "The Lighting Handbook, 10th Edition". Illuminating Engineering Society. USA.
Doulos, L., Tsangrassoulis, A. \& Topalis, F. V. (2005) A critical review of simulation techniques for daylight responsive systems. Proceedings from the European Conference on Dynamic Analysis, Simulation and Testing applied to the Energy and Environmental performance of Buildings DYNASTEE, Athens (EL), 12-14 October 2005

Doulos, L., Tsangrassoulis, A. and Topalis, F. (2008a). Quantifying energy savings in daylight responsive systems: The role of dimming electronic ballasts. Energy and Buildings, 40(1), 36-50.

Doulos, L., Tsangrassoulis, A. and Topalis, F. V. (2008b). The role of spectral response of photosensors in daylight responsive systems. Energy and Buildings, 40(4), 588-599.

Dubois, M. C. and Blomsterberg, Å. (2011). Energy saving potential and strategies for electric lighting in future North European, low energy office buildings: A literature review. Energy and Buildings, 43(10), 2572-2582.

Ehrlich, C., Papamichael, K., Lai, J., \& Revzan, K. (2002). A method for simulating the performance of photosensor-based lighting controls. Energy and buildings, 34(9), 883-889.

European Committee for Standardisation (2011). Light and lighting - Lighting of work places - Part 1: Indoor work places ( EN 12464-1).

European Committee for Standardisation (2017). Energy performance of buildings - Energy requirements for lighting (EN 15193-1).

Littlefair, P. J. (2001). Photoelectric control: the effectiveness of techniques to reduce switching frequency. Lighting Research and Technology, 33(1), 43-55.

Lo Verso, V. R. M., Pellegrino, A. and Pellerey, F. (2014). A multivariate non-linear regression model to predict the energy demand for lighting in rooms with different architectural features and lighting control systems. Energy and Buildings, 76, 151-163.

Reinhart, C. F. (2004). Lightswitch-2002: a model for manual and automated control of electric lighting and blinds. Solar Energy, 77(1), 15-28.

Reinhart, C. F. (2006). Tutorial on the use of daysim simulations for sustainable design. Institute for Research in Construction, National Research Council Canada. Ottawa (Ont.).

Rogers, Z. (2006). Daylighting Metric Development Using Daylight Autonomy Calculations. Sensor Placement Optimization Tool. Boulder, Colorado, USA: Architectural Energy Corporation.

Roisin, B., Bodart, M., Deneyer, A., \& D'herdt, P. (2008). Lighting energy savings in offices using different control systems and their real consumption. Energy and Buildings, 40(4), 514-523. 